\title{
Maternal Perceptions of Cesarean Delivery Care: A Qualitative Study to inform ERAS Guideline Development
}

Krista Wollny ( $\square$ krista.wollny@ucalgary.ca )

University of Calgary https://orcid.org/0000-0002-7709-1030

Amy Metcalfe

Alberta Health Services

Crystal Corrigan

Alberta Health Services

Ashley Drobot

Alberta Health Services

Loreen Gilmour

University of Calgary

Stephen Wood

University of Calgary

R. Douglas Wilson

University of Calgary

Leah Gramlich

University of Alberta

Gregg Nelson

University of Calgary

Research article

Keywords: cesarean delivery, qualitative research, ERAS, guidelines

Posted Date: November 7th, 2019

DOI: https://doi.org/10.21203/rs.2.16977/v1

License: (c) (1) This work is licensed under a Creative Commons Attribution 4.0 International License.

Read Full License 


\section{Abstract}

INTRODUCTION: Cesarean delivery (CD) is the most common inpatient surgical procedure; however, there are no internationally-accepted, standardized clinical guidelines. The Enhanced Recovery After Surgery (ERAS $\left.{ }^{\circledR}\right)$ program aims to improve outcomes through the development of international guidelines (IG). The purpose of this study is to obtain and consolidate women's experiences with CD to inform the development of an ERAS international guideline (IG).

METHODS: Qualitative methods were used to assess the patient experience with current evidence-based $C D$ protocols across operative phases. Twelve women who experienced $C D$ were interviewed using an open-ended, semi-structured interview guide at six-weeks post-partum. Two researchers coded the emerging themes separately and compared findings.

RESULTS: Women described feeling informed, but felt they did not have a choice. Pre-surgery, women wanted more information about the risks of CD. Pre-operatively, women expressed confusion with the procedures, but felt informed about local anaesthesia and thermoregulation. Post-CD, women felt informed about pain and nausea control; however, urinary catheter removal was delayed when compared to the ERAS recommendations. Information about post-partum infant care was not well communicated, as many women were uninformed about delayed cord-clamping and infant thermoregulation.

DISCUSSION: This qualitative study provides opportunities to improve communication, the patientprovider relationship, and the overall satisfaction throughout the CD process. The findings support the implementation of patient decision aids and training with the shared decision model. The improved procedures recommended in the ERAS IG for CD have the potential to deliver significant improvements to patient satisfaction, mother-infant bonding and mental wellness.

\section{Background}

Cesarean delivery (CD) is the surgical delivery of a neonate through the abdominal and uterine wall, performed for indications of poor maternal and fetal physiology such as labour dystocia, fetal distress, and fetal presentation $(1,2)$. It is the most common inpatient surgical procedure around the world (Canadian Institute for Health Information, n.d.; Kozhimannil, Law, \& Virnig, 2013). CD has additional risks and complications for women and newborns and is not a routine recommendation for all births (Mazzoni et al., 2011). Currently there are no internationally accepted standardized guidelines for CD clinical protocols. Professional societies provide clinical recommendations for surgical interventions, but healthcare organizations and institutions are responsible for creating policies and procedures to implement and oversee obstetrical processes and services for CD deliveries. Interpretation and implementation of these protocols and procedures vary. Standardizing the processes and procedures towards patients' enhanced care and recovery is fundamental to reduce protocol variance and to improve CD outcomes. 
When developing enhanced pregnancy clinical pathways, the process must consider women's preferences for mode of delivery and provide the information required to make informed choices regarding mode of delivery (Mazzoni et al., 2011; Mazzoni et al., 2016; Torloni et al., 2013; Yee et al., 2015). Time to counsel and educate the patient is a major obstacle to protocol driven quality improvement for clinicians. A positive delivery experience can result in a sense of accomplishment and feelings of self-worth and selfconfidence for the woman; however, a negative delivery experience can result in feelings of maternal distress, postpartum depression and post-traumatic stress disorder (9). Recent studies suggest women undergoing scheduled $C D$ have higher satisfaction ratings than women undergoing vaginal delivery $(10,11)$. Professional associations, such as the World Health Organization, however, warn that the increasing number of scheduled CD in industrialized countries generate supplementary costs and carry greater health risks for the woman and child (9). Consequently, as guidelines and clinical pathways are standardized, experiences of women must be integrated to ensure optimal CD-related care.

The Enhanced Recovery After Surgery (ERAS $\left.{ }^{\circledR}\right)$ Program is a novel approach to improve patient-focus and outcomes. ERAS programs and guidelines are developed internationally using standardized methodology (12). The surgery-specific programs include an evidence-based guideline, a knowledge translation implementation plan, and a data collection and audit system (Gustafsson et al., 2013; Gustafsson, 2011). A critical component in the development of an ERAS international guideline is the patient voice. A qualitative study of women's experiences with $C D$ is necessary to clarify considerations for guideline creation and implementation to improve patient satisfaction. The purpose of this study is to obtain and consolidate these maternal patient experiences to inform the development and implementation of an ERAS international guideline (IG) for CD.

\section{Methods}

Qualitative methods were used to assess the patient experience with current evidence-based CD protocols across operative phases: pre-surgery maternal care, pre-operative procedures, post $C D$, and post-partum infant care, to inform the development of ERAS IG for CD. Participants were recruited through posters and postcards in the post-surgery follow-up clinic at a tertiary care hospital in Calgary, Alberta. Inclusion criteria included women who underwent a scheduled CD for their most recent delivery. The women were interviewed six weeks post-partum between May to October 2018. This study was approved by the Conjoint Health Research Ethics Board at the University of Calgary. Prior to the interview, consent was obtained after the participants were informed of the purpose of the study, any potential harms and benefits of participating, and that all information obtained would be confidential. Participants consented to the interview being audio-recorded.

Open-ended, semi-structured interviews were conducted in-person and over the phone at six weeks postpartum by a licensed practical nurse. The nurse began by explaining that a cesarean delivery is a complex process and the guidelines are being updated to reflect new knowledge about how to best support moms and babies (see Appendix for the semi-structured interview guide). Participants were 
reminded that participation was optional, and they were not obligated to respond to any questions they were not comfortable answering.

Interview questions were asked according to the operative phases, starting with pre-surgery. All questions asked reflected the updated, evidence-based recommendations for CD. Participants were asked if their physician discussed certain issues with them prior to being admitted to the hospital, such as informed consent for the $\mathrm{CD}$, cigarette smoking, and chronic conditions including obesity and hypertension. The questions about the pre-operative procedures asked about prophylactic antibiotics, anaesthesia, fasting, and thermoregulation. The questions about the post-CD experience asked about the anti-nausea and pain control medications that were offered, when the urinary catheter was removed, and if the women were encouraged to move, walk, and begin eating a regular diet. The last phase the participants were questioned about was the care provided to the baby during and after birth, such as delayed cordclamping, infant temperature monitoring, suctioning, and oxygen use. When the guided questions were asked about the above phases, the participants were also asked to provide advice to the healthcare team that would allow future women to receive the best care possible.

Interviews ranged from 10-15 minutes, and were audio recorded for transcription purposes. An initial 4 interviews were conducted and transcribed as a pilot. Following this, a further 8 interviews were conducted and transcribed. The emerging themes were coded separately and then compared by two researchers $(C C, A D)$ with graduate-level training in qualitative analysis. All twelve interview transcripts were analysed using NVivo software.

\section{Results}

\section{Major findings}

Women felt informed, but felt like they did not have a choice.

Most of the women (9 out of 12) indicated that they felt informed throughout the CD process (from presurgery to post-partum infant care). However, despite feeling informed, seven of the women indicated feeling as though they did not have a choice in decision making, or that their voices were not heard. This was mainly described in relation to pre-surgical obstetric care, including anesthesia procedures, catheter removal, scheduling pain medication, cord camping, and the application of erythromycin eye-drops to the baby.

"With a C-section I feel like I just didn't have a choice, and this time around they said this is what happens, and that was it." Interview 5 
When women compared the current CD to previous births, more positive responses towards the current birth were elicited when women felt involved in decision making (4/6). Women also described positive experiences in relation to: being informed stepwise about procedures, being kept comfortable, and staying warm and pain free.

\section{Advice from Women}

Four of the twelve women offered advice on ways to improve the care they received during their CD. This included sharing information specific to the risks and harms of procedures sooner, improving communication during the surgery and birth process, having more experienced nurses on shift, and having the opportunity to bond skin-to-skin with the baby immediately after birth. Other suggestions from women involved the food that was or was not available outside of mealtimes, and having alternatives to toast (a common post-surgical offering) for women with gluten restrictions.

\section{Findings by phase:}

\section{Pre-surgery Maternal Care}

The ERAS IG for CD recommends that an antenatal optimized pathway begins at 10-20 weeks gestation to support preadmission information, patient education and counselling on maternal comorbidities (15).The majority of women who participated in this study reported feeling well informed; however, when asked in detail, many of the pre-surgical risks recommended for discussion may not have been discussed, or were identified as being not applicable to their experience, such as anaemia, diabetes control, or smoking cessation.

Few women reported experiencing complications, and the majority of women who did experience complications were informed of the risks, treatments, and interventions associated with those conditions. For example, three women experienced anaemia treatment and interventions. Of those, two described being explicitly informed about anaemia in pregnancy. One woman smoked and was counselled on the impact of smoking. Additionally, some women were informed of potential complications that they did not experience. For example, two women experienced gestational diabetes, and seven indicated they were informed about the condition.

When discussing their experiences with pre-surgery or primary obstetric care phase, several women described wanting to be more informed about the risks prior to delivery, regardless of previous CD experience. 
"The day of... was overwhelming for me... they went over possibilities of getting an infection and that kind of thing and the risks... I think the timing of that maybe could've been...before [the day of the procedure]..." Interview 5

\section{Pre-Operative Procedures}

The ERAS IG for CD provide specific recommendations for pre-operative CD care, including anesthetic medications, fasting, antimicrobial prophylaxis, and thermoregulation (15).

Five women expressed confusion with procedures during the pre-operative phase as well as limited time to clarify concerns. Of those who experienced confusion, three women with prior births by CD indicated this was because little detail in the current pregnancy was provided, as it was assumed that the women would know what to expect. However, one woman did indicate the communication and description of the processes was better at the current site as compared to another hospital where she had delivered by CD in the past.

"...they didn't go into a lot of great detail...[and] this was not my first $C$-section..." Interview 6

In preparation for the surgery, patient-provider discussions about local anesthesia (12/12), being kept warm (11/12), and having an understanding of provision of antibiotics were most commonly experienced (3/12). Of those receiving local anesthesia, eight indicated they were explicitly informed of the process. It could not be determined if the women were informed why local anesthesia was preferred over general sedation. With respect to the anesthetic process, however, of those who referenced positive interactions with health professionals overall (8/12), three stated a positive experience with the anesthetist specifically.

Only two women experienced heartburn, constipation (prior to surgery), or medication concerns. One indicated the discussion around heartburn medications left her feeling informed of the implications. No women experienced pre-operative oral or manual bowel preparations or were informed of the recommendation to avoid bowel preparations. Interestingly, nine women fasted (no oral intake of solid food) longer than the recommended six hours prior to elective surgery (15). One woman explicitly stated waiting six hours, and the other two responses were vague with regards to timing. Nine women indicated they were instructed to fast from 8-12 hours prior to the surgery.

Post CD 
The ERAS IG for CD provide specific recommendations for post-operative CD care, including nausea and pain control, urinary catheter removal, and mobilization (16). Post-surgery, pain was well managed and medication provision was effectively communicated by the nursing staff. All (12/12) women indicated adequate pain control, and most (11/12) reported being explicitly informed of the type, reasoning, or scheduling of the medications. One woman remained unsure of the types of medications she was provided and indicated discussion occurred primarily at discharge around types and timing of pain control medications.

The ERAS IG for CD recommends immediate removal of urinary catheter after CD to prevent infection and increase mobility (16); however, eleven of the twelve women did not have the catheter removed immediately after surgery. When timing was offered, removal occurred greater than 12 hours later, in the range of 12-36 hours post-CD. Three women suggested they did not have a choice as to when the catheter could be removed. Two explicitly requested catheter removal, whereas seven of the twelve women had it removed by nursing at varying times, with some women waiting for a shift change. Women waiting up to 36 hours for catheter removal suggested they were not informed it could come out earlier. Early mobilization was often referenced as a point of catheter removal; however, only one of those who mobilized early also had the catheter removed, and nine of the twelve women were moving as soon as possible. Of those moving soon after the procedure, two stated explicitly they were encouraged to get up. Of the three who were not moving as soon as possible, one was encouraged, but not soon afterwards, while the second woman informed the nursing staff instead, due to her occupation as a physiotherapist.

"...it wasn't able to come out until I was able to get up and walk around myself, so it was, l'd say maybe like 36 hours after..." Interview 3

The most expressed area of anxiety was the inability to eat post-surgery, particularly for the women who did not have someone available to get food for them outside of meal times. Some women indicated they were instructed not to eat after their $C D$, while others described food not being available outside of the hospital's meal times. In all, eight of the women did not eat a regular diet within two hours of the procedure as recommended by the ERAS IG for CD (16). When offered, women indicated they had fasted between a total of $18-30$ hours.

"...food was ...stressful for me on that first day because I was really hungry and ...if anybody is without support, like I had my husband to go over and get stuff for me but ... he was in the NICU ... for quite some time first ... when he came out I was...starving..." Interview 1 
The ERAS IG for CD provide specific recommendations for post-partum infant care, including delayed cord clamping, thermoregulation, avoiding routine airway suctioning, and avoiding supplemental oxygen therapy if possible (17). Of the operative phases explored, being informed of infant related procedures was most often described as absent. The majority of women were not informed if their infant had experienced the recommendations described above. None of the women were informed that extra oxygen is not recommended unless resuscitation was required. Nine women were unaware of the delayed cord clamping of one minute for full-term infants and 30 seconds for preterm infants. Most (11/12) expressed they did not know when cord clamping occurred; one woman was aware when it occurred but was uncertain about the timing of clamping.

One woman was explicitly told suctioning may happen as meconium was present in the womb, whereas ten others were neither aware nor explicitly informed. One woman requested her infant be suctioned and another noticed her infant was suctioned; however, she was not informed that it was not a routine practice unless the airway is obstructed.

With regards to temperature control and the recommendation to monitor the infant's temperature, three women were explicitly informed. Nine women were neither informed of the recommendation nor aware of whether this was being done. Of those, one woman overheard it was happening and one woman was able to see hospital staff do it; however, conversations in the room did not include these women. Overall, women were unaware of the processes or procedures performed immediately after the birth of their child.

"...my husband could see it, and then I could see it......so I was aware of it happening" (Referring to temperature monitoring without explicitly being informed) Interview 8

\section{Discussion}

This qualitative study provides opportunities to improve communication, the patient-provider relationship, and the overall satisfaction throughout the CD process. The CD process is not simply a surgery; it is a means of childbirth, which many women view as a landmark event (9). For some women, delivering via $\mathrm{CD}$ may equate to an inability to be involved in decision making. For women with the lowest perceived involvement, the risk for post-partum depression is the greatest $(18,19)$. As a systematic review of patient satisfaction suggests, effective communication and the patient-provider relationship is integral to improved experience (20). Two-way discussions and shared decision making may help to improve this.

Shared decision making is a process in which clinicians and patients communicate choices about screening, treatment, and intervention together. It is central to quality care. Shared decision making in maternity care has potential to reduce decisional conflict, increase satisfaction with the delivery experience, and reduce depressive symptoms (21). Porter et al. (2007) examined a survey of over 1600 
women to identify distressing factors related to the CD process. Women listed poor communication, fear of missing out on a natural birth, and the immediate post-partum experience as the most distressing (22). Melman et al. (23) also found practitioner hesitation to allow women to be part of the decision making process as a common barrier to delivering optimal CD care. Women's opinions and experiences, however, are a necessary consideration in the CD process. Not surprisingly, the proportion of patients reporting a desire to participate in shared decision making is increasing (24).

As with all procedures, explaining the sequence of events and expectations beforehand is vital to decreasing distress (19). In the pre-operative phase, women described being told the processes were "just the way it was done," with little room for discussion. Clearly communicating the steps in the surgical processes may improve patient perception of control, involvement, and further improve patient satisfaction.

In line with findings from Porter et al.'s (22) research, women most often referenced feelings of not having a choice immediately post-surgery. In our sample, all the women were able to verbalize concerns regarding catheter removal and fasting post-surgery. However, as Annastasio et al. (24) pointed out, if the woman had low educational attainment, low socio-economic status, or for whom English was not their first language, the ability to communicate concerns and the feelings of shared decision making is greatly reduced.

Women were similarly ill-informed or unaware of events during post-partum infant care and recommended improved communication. Feeling uninformed when experiencing a first CD negatively affects mental wellbeing (22). Along with improved communication during the $C D$, having the opportunity to bond skin-to-skin with the baby immediately after birth was identified as important when women felt a loss of control over the birthing process. Despite research supporting early mother-infant contact, CD delivery practices have not reformed greatly over the past 30 years (25).

This study is not without limitations. For more robust and consistent data, opportunities exist for refinement of the interview guide. Inclusion of exploratory probes may further assist in triangulation with secondary data sources. Examples might include reasons for CD, previous birth experiences, and explicitly exploring recommendations with regards to shared decision making. Adding patient satisfaction related surveys and focus groups with varied demographics, locations, and clinical circumstances may also allow for improved implementation and understanding of varied contexts. Interview data were collected and analyzed by different team members, leaving context and nuances difficult to detect. Additionally, responses did not consistently indicate whether the woman was informed about the implications of a condition on the pregnancy or whether she simply experienced the condition. For example, one woman may have experienced hypertension, but may not have been informed of the implications. This caused difficultly for analysis when attempting to discern if the woman was informed and involved in decision making. The small sample size and absent demographic and contextual variables prevented robust analysis of variance.For more robust findings, particularly in higher risk 
populations, additional investigations around communication and shared decision making are suggested to enhance recommendations for improved patient experience.

\section{Conclusions}

The findings from this study, along with evidence from the literature, support the implementation of novel strategies including patient decision aids and training with the shared decision model. Structured implementation of decision aids specific to each phase of the process is recommended to ensure true informed consent. Implementation of decision aids and training of providers must also be attentive to the needs of vulnerable groups to ensure such interventions reduce rather than widen disparities (24). Suggestions for further investigations include examination of the outcomes of implementing patient decision aids and shared decision-making training. This will enhance the data available about how the cesarean procedures or guideline implementation relates to a woman's involvement in decision making or sense of control and choice (24). Once the data are holistically examined, and the creation and implementation of the ERAS CD guidelines has occurred, the improved procedures have the potential to deliver significant improvements not only to patient satisfaction, but also to mother-infant bonding and mental wellness.

In order for ERAS CD guidelines to improve standardization of surgical procedures, enhance recovery and optimize patient outcomes, a woman's experience with shared decision making throughout the operative phases must be considered. In the context of rising CD rates throughout the developed world, and as international guidelines are crafted, doing so will help to focus efforts and respond to the patient voice.

\section{List Of Abbreviations}

CD - Cesarean delivery (CD)

ERAS - Enhanced Recovery After Surgery ${ }^{\circledR}$

IG - International Guidelines

\section{Declarations}

\section{Ethics Approval and Consent to Participate}

This study was approved by the Conjoint Health Research Ethics Board at the University of Calgary. Prior to the interview, consent was obtained after the participants were informed of the purpose of the study, any potential harms and benefits of participating, and that all information obtained would be confidential. Participants consented to the interview being audio-recorded.

\section{Consent for Publication}

Not applicable. 
Availability of Data and Materials

Not applicable.

\section{Competing Interests}

The authors declare that they have no competing interests.

\section{Funding}

Funding for this project was from a University of Calgary Seed Grant. The funding body did not contribute to the design of the study, data collection, analysis, interpretation of data, or writing of the manuscript.

\section{Authors' Contributions}

All authors made a substantial contribution to this study. AM, LG, SW, RDW, LG, and GN designed the study; $C C$ and $A D$ conducted the analysis, $\mathrm{KW}$ drafted the manuscript. All authors jointly interpreted the findings, critically reviewed the manuscript, and approved the final version for submission.

\section{Acknowledgements}

We would like to thank the women who shared their experiences with us, contributing to the development and implementation of a patient-informed guideline.

\section{References}

1. Kozhimannil KB, Arcaya MC, Subramanian SV. Maternal Clinical Diagnoses and Hospital Variation in the Risk of Cesarean Delivery: Analyses of a National US Hospital Discharge Database. Smith GC, editor. PLoS Med. 2014 Oct 21;11(10):e1001745.

2. Debas HT, Donkor P, Gawande A, Jamison DT, Kruk ME, Mock CN, editors. Disease Control Priorities, Third Edition (Volume 1): Essential Surgery [Internet]. The World Bank; 2015 [cited 2019 Apr 29]. Available from: http://elibrary.worldbank.org/doi/book/10.1596/978-1-4648-0346-8. Accessed 29 Apr 2019.

3. Canadian Institute for Health Information. Hospital Morbidity Database.

4. Kozhimannil KB, Law MR, Virnig BA. Cesarean delivery rates vary tenfold among US hospitals; Reducing variation may address quality and cost issues. Health Aff (Millwood). 2013;32(3):527-35.

5. Mazzoni A, Althabe F, Liu N, Bonotti A, Gibbons L, Sánchez A, et al. Women's preference for caesarean section: a systematic review and meta-analysis of observational studies: Women's preference for caesarean section: systematic review. BJOG Int J Obstet Gynaecol. 2011 Mar;118(4):391-9.

6. Mazzoni A, Althabe F, Gutierrez L, Gibbons L, Liu NH, Bonotti AM, et al. Women's preferences and mode of delivery in public and private hospitals: a prospective cohort study. BMC Pregnancy 
Childbirth. 2016 Dec;16(1):34.

7. Torloni MR, Betrán AP, Montilla P, Scolaro E, Seuc A, Mazzoni A, et al. Do Italian women prefer cesarean section? Results from a survey on mode of delivery preferences. BMC Pregnancy Childbirth. 2013 Dec;13(1):78.

8. Yee LM, Kaimal AJ, Houston KA, Wu E, Thiet M-P, Nakagawa S, et al. Mode of delivery preferences in a diverse population of pregnant women. Am J Obstet Gynecol. 2015 Mar;212(3):377.e1-377.e24.

9. Guittier M-J, Cedraschi C, Jamei N, Boulvain M, Guillemin F. Impact of mode of delivery on the birth experience in first-time mothers: a qualitative study. BMC Pregnancy Childbirth. 2014 Dec;14(1):254.

10. Wiklund I, Edman G, Andolf E. Cesarean section on maternal request: reasons for the request, selfestimated health, expectations, experience of birth and signs of depression among first-time mothers. Acta Obstet Gynecol Scand. 2007;86(4):451-6.

11. Blomquist J, Quiroz L, MacMillan D, Mccullough A, Handa V. Mothers' Satisfaction with Planned Vaginal and Planned Cesarean Birth. Am J Perinatol. 2011 May;28(05):383-8.

12. ERAS Society. ERAS Society Policy on Guidelines [Internet]. Available from: http://erassociety.org/guidelines/policy-on-guidelines/. Accessed 14 May 2019.

13. Gustafsson UO. Adherence to the Enhanced Recovery After Surgery Protocol and Outcomes After Colorectal Cancer Surgery. Arch Surg. 2011 May 1;146(5):571.

14. Gustafsson UO, Scott MJ, Schwenk W, Demartines N, Roulin D, Francis N, et al. Guidelines for Perioperative Care in Elective Colonic Surgery: Enhanced Recovery After Surgery (ERAS®) Society Recommendations. World J Surg. 2013 Feb;37(2):259-84.

15. Wilson RD, Caughey AB, Wood SL, Macones GA, Wrench IJ, Huang J, et al. Guidelines for Antenatal and Preoperative care in Cesarean Delivery: Enhanced Recovery After Surgery Society Recommendations (Part 1). Am J Obstet Gynecol. 2018 Dec;219(6):523.e1-523.e15.

16. Macones GA, Caughey AB, Wood SL, Wrench IJ, Huang J, Norman M, et al. Guidelines for Postoperative care in Cesarean Delivery: Enhanced Recovery After Surgery (ERAS) Society Recommendations (Part 3). Am J Obstet Gynecol. 2019 Apr;S0002937819305721.

17. Caughey AB, Wood SL, Macones GA, Wrench IJ, Huang J, Norman M, et al. Guidelines for intraoperative care in cesarean delivery: Enhanced Recovery After Surgery Society Recommendations (Part 2). Am J Obstet Gynecol. 2018 Dec;219(6):533-44.

18. Goodman P, Mackey MC, Tavakoli AS. Factors related to childbirth satisfaction. J Adv Nurs. 2004 Apr;46(2):212-9.

19. Burcher P, Cheyney MJ, Li KN, Hushmendy S, Kiley KC. Cesarean Birth Regret and Dissatisfaction: A Qualitative Approach. Birth. 2016 Dec;43(4):346-52.

20. Crow R, Gage H, Hampson S, Hart J, Kimber A, Storey L, et al. The measurement of satisfaction with healthcare: implications for practice from a systematic review of the literature. Health Technol Assess Winch Engl. 2002;6(32):1-244.

21. Goldberg H. Informed Decision Making in Maternity Care. J Perinat Educ. 2009 Jan 1;18(1):32-40. 
22. Porter M, van Teijlingen E, Chi Ying Yip L, Bhattacharya S. Satisfaction with cesarean section: qualitative analysis of open-ended questions in a large postal survey. Birth Berkeley Calif. 2007 Jun;34(2):148-54.

23. Melman S, Schreurs RHP, Dirksen CD, Kwee A, Nijhuis JG, Smeets NAC, et al. Identification of barriers and facilitators for optimal cesarean section care: perspective of professionals. BMC Pregnancy Childbirth. 2017 Dec;17(1):230.

24. Attanasio LB, Kozhimannil KB, Kjerulff KH. Factors influencing women's perceptions of shared decision making during labor and delivery: Results from a large-scale cohort study of first childbirth. Patient Educ Couns. 2018 Jun;101(6):1130-6.

25. Magee SR, Battle C, Morton J, Nothnagle M. Promotion of Family-Centered Birth With Gentle Cesarean Delivery. J Am Board Fam Med. 2014 Sep 1;27(5):690-3.

\section{Appendix - Semi-structured Interview Guide}

\section{Background, objectives}

-Thank participant for time; Review consent form and ensure signatures in place -Review: all input is valued, all input is confidential; may I tape conversation

\section{Proposed new Guidelines (which patient participates in):}

Questions will be asked in terms of: Cesarean delivery is a complex process and the guidelines are being updated to reflect new knowledge about how to best support moms and babies. We are interested in your experience with the following procedures or practices. You may not have experienced many of these areas that we're asking about so we can skip any that don't apply or if you don't want to discuss them.

\section{Pre-Surgery. The first group of questions are about your experience before you went to the hospital for your procedure. We're interested in whether your doctor discussed these issues with you and what advice you have so that the best care can be provided?}

a. Informing the patient before, during and after all procedures including a preadmission discussion of the harms and benefits to both mother and baby.

b. Cigarette smoking stopped

c. Maternal obesity of bmi $>40$

d. Anemia (low red blood count) should be identified and corrected

e. Chronic hypertension

f. Gestational Diabetes 
II. Pre-operative Procedures. The next set of questions are related to your experience just before the surgery and they reflect the new recommendations for Cesarean delivery. We're interested in your experience and what advice you may have so others get this care.
a. Antibiotics 60 minutes before
b. General Sedation avoided; local anesthesia is preferred
c. Heart burn or constipation concerns or medication experience?
d. Bowel preps should not be administered
e. Fasting: clear fluids up to 2 hours before and light meal up to 6 hours prior
f. Keep patient warm

\section{Post Cesarean delivery. The next set of questions are about your experience after your surgery and again reflect the new recommendations. We're interested in your experience and your advice so that others get this care.}
a. Begin regular diet 2 hours after
b. Anti-nausea meds and procedures are available
c. Pain control meds are recommended
d. Catheter removal immediately after procedure
e. Moving and walking as soon as possible after procedure

IV. Your baby. The last set of questions are about your baby and again reflect the new recommendations. What was your experience and what is your advice?
a. Clamping the cord at least 1 minute after delivery for a full term baby and 30 seconds after for a preterm baby.
b. The baby's body temperature should be monitored
c. Suctioning of the baby's airway should only be done if there appears to be something blocking it; not a routine procedure.
d. Extra oxygen should be avoided unless resuscitation is needed. 
Ask if there any other issues of concern.

Give the pre-paid gift card and sign the budget sheet acknowledging receiving the gift card. 